\title{
V a \\ Monitoring des Beitrags der Psychologie zur interdisziplinären Bildungsforschung: Szientometrische Analysen zur Forschung aus dem deutschsprachigen im Vergleich zum angloamerikanischen Bereich
}

Günter Krampen, University of Trier

Marcus Hasselhorn, German Institute for International Educational Research DIPF, Frankfurt am Main

In einer disziplinspezifischen Perspektive wird szientometrisch dem Beitrag psychologischer Fachpublikationen zur Bildungsforschung nachgegangen. Notwendig ist diese Einschränkung, da die Entwicklung der Publikationszahlen zur psychologischen Bildungsforschung insgesamt und zur Teilmenge der empirischen Bildungsforschung aus dem deutschsprachigen Bereich mit denen angloamerikanischer Publikationen verglichen werden. Dazu sind Angaben zur AutorInnen-Affiliation notwendig, die in vielen Fachdatenbanken nicht oder nur selektiv, in denen für die Psychologie (PSYNDEX, PsycINFO) jedoch durchgängig vorhanden sind. Die Befunde beschreiben die Entwicklung des psychologischen Publikationsaufkommens zur Bildungsforschung und zu der Teilmenge der empirischen Bildungsforschung (a) im deutschsprachigen und (b) angloamerikanischen Bereich sowie (c) deren Vergleiche. Abgebildet wird das Publikationsaufkommen von 1945 bis 2014 und in Feinanalysen von 1980 bis 2014.

\section{Einleitung}

Bildungsforschung ist interdisziplinär angelegt und bündelt bei der Erforschung komplexer formeller (institutioneller) und informeller (gesellschaftlicher) Bildungs- und Erziehungsprozesse in der Lebensspanne synergetisch Kompetenzen aus vielen wissenschaftlichen Disziplinen (vgl. etwa Tippelt \& Schmidt, 2010). Dazu gehören vor allem die Erziehungswissenschaften, Fach-Didaktik, Geschichtswissenschaft, Ökonomie, Philosophie, Politikwissenschaft, Psychologie, Rechtswissenschaft, Soziologie, Informationswissenschaften und Neurowissenschaften. Die Forschungserträge der Bildungsforschung werden sowohl in den Fachmedien der einzelnen Wissenschaften als auch - seit einigen Dekaden - in eigenen interdisziplinär orientierten Medien für die Bildungsforschung und 
Bildungswissenschaften breit gestreut publiziert und dokumentiert. Dies wurde z. B. von Kuhberg-Lasson, Singleton und Sondergeld (2014) anhand von Informationen aus disziplinspezifischen Fachdatenbanken der Bildungs- und Erziehungswissenschaften (FIS Bildung), Psychologie (PSYNDEX) und Sozialwissenschaften (SOLIS) beschrieben. Kuhberg-Lasson et al. konzentrieren sich dabei auf die formalen Charakteristika der Publikationen aus Forschungsprojekten der Bildungsforschung.

Ebenso wie bei Knaupp, Schaufler, Hofbauer und Keiner (2014) zeigten sich bei Kuhberg-Lasson et al. (2014) markante Unterschiede in den fachspezifischen Publikationskulturen. Diese beziehen sich vor allem auf

- die Publikationsarten (wie Präferenz für Journal-Beiträge, Monographien und/oder Buchbeiträge),

- die Anzahl und den Umfang von Ko-Autorenschaften,

- die Anzahl der Publikationen aus einzelnen Forschungsprojekten,

- das Ob und das Wie von peer reviews der zur Publikation eingereichten Manuskripte,

- die Internationalität der Publikationen, also die Publikationssprache.

Die Unterschiede zwischen den an der Bildungsforschung beteiligten disziplinären Publikationskulturen betreffen damit sowohl qualitative als auch - in der Mehrzahl - quantitative Kriterien des publizierten Forschungsertrages. Knaupp et al. (2014, S. 107) ziehen aus ihren Vergleichen von Fachzeitschriften der Erziehungswissenschaft versus Pädagogischen Psychologie aus Deutschland, Grossbritannien und Italien das «vorläufige» Fazit, «dass sich unterschiedliche Kommunikationsmuster sowohl hinsichtlich disziplinärer wie wissenschaftskultureller Merkmale feststellen lassen.»

\section{Szientometrischer Zugang}

Die Soziometrie ist eine quantitative Methode, keine Taxonomie oder Theorie der Wissenschaftsforschung (siehe etwa Felt, Nowotny \& Taschwer, 1995; Krampen \& Montada, 2002). Die eigentlichen und auch die ursprünglichen Anwendungen der Szientometrie beziehen sich auf die Beschreibung von Forschungs- bzw. Publikationsschwerpunkten sowie -lücken in den Wissenschaften sowie auf die darauf bezogenen historischen Entwicklungstrends. Genutzt wurden und werden grosse Datensätze (big data) zu wissenschaftlichen Publikationen, Forschungsprojekten, Kongressen, Fachtagungen etc., die in ihrer schieren, in der zweiten Hälfte des 20. Jahrhunderts stark angestiegenen, Menge von Einzelnen nicht umfassend rezeptiert werden können. Bis in die 1980er Jahre wurde dies mühsam anhand von Print-Medien in quantitativen Inhaltsanalysen von Kongress- und Tagungsberichten, Abstract-Diensten, Fachzeitschriften, Büchern etc. umgesetzt. Durch die Digitalisierung stehen inzwischen 
im Internet äusserst umfangreiche Datenbanken zur Verfügung, in denen diese Informationen für einzelne wissenschaftliche Disziplinen (Fachdatenbanken) oder fachübergreifend enthalten sind. Mit differenzierten Recherchestrategien werden dadurch umfassendere deskriptive szientometrische Inhaltsanalysen möglich, was in einigen dieser Datenbanken durch Metadaten zu den einzelnen Dokumenten (etwa systematische Inhalts-Klassifikationen mit Feinauflösungen und/oder Schlagworte nach einem dynamischen Thesaurus) erleichtert wird.

Von dieser deskriptiven Anwendung der Szientometrie ist ihre mit der Digitalisierung und der Verfügbarkeit der Datenbanken im Internet verstärkt aufgekommene normative Verwendung zu Evaluationszwecken streng zu unterscheiden. Diese normative Anwendung bezieht sich auf Versuche, wissenschaftliche Arbeitsleistungen einzelner Forscherinnen bzw. Forscher, Departments, Universitäten oder Forschungsinstitute, ganzer Regionen und/oder Nationen komparativ zu evaluieren. Beliebt in Wissenschaftsmanagement und -politik ist dieser Ansatz in den Wissenschaften selbst aufgrund zahlreicher methodischer Probleme umstritten (vgl. im Überblick etwa Krampen, 2013).

Die quantitative Szientometrie hat auch bei ihren deskriptiven, wissenschaftliche Entwicklungstrends beschreibenden Anwendungen ebenso wie alle anderen Forschungsmethoden spezifische Vor- und Nachteile, weist also auch Beschränkungen auf. Quantitative Methoden werden nach wie vor oftmals gerade auch im Kontext der Bildungsforschung - qualitativen Ansätzen entgegengestellt. Dies wurde bereits von Bühler (1927) für das Gegenstands- und Methodenverständnis der Psychologie abgelehnt. Bühler folgend hat dies Elsa Köhler (1929) für die Pädagogik zugunsten einer aus dem Gegenstand dieser Wissenschaften abzuleitenden Notwendigkeit der methodologischen Integration ausgeführt. Entsprechendes gilt auch für die Beschreibung und das Monitoring von Forschungserträgen (als von Menschen erzeugte «Werke» sensu Bühler, 1927, S. 64), die etwa in Form von Publikationen gezählt werden können. So können Forschungsschwerpunkte und -entwicklungen beschrieben werden, die aber zusätzlich der fachlichen, inhaltlichen und gesellschaftlichen, also der qualitativen Deutung und Bewertung bedürfen.

Bei bewilligten Forschungsanträgen und publizierten Fachbeiträgen, die einem peer review unterzogen wurden, wurde eine wissenschaftliche, also qualitative Bewertung von dem, was dann szientometrisch quantifiziert wird, vorab vorgenommen. Wegen der grossen, von Einzelnen kaum überblick- und rezipierbaren Anzahl wissenschaftlicher Publikationen in ganzen Forschungsbereichen sind szientometrische Quantifizierungen beim Monitoring ein sinnvolles und nützliches Hilfsmittel für später folgende Qualifizierungen, d.h. der zusammenfassenden Deutung und Bewertung ihres wissenschaftlichen und gesellschaftlichen Ertrags (siehe hierzu Weiteres bei Krampen, 2013).

Der Einsatz der Szientometrie beim komparativen Monitoring der Forschungsentwicklung in unterschiedlichen Forschungsgemeinschaften kann darüber hinaus die fachinterne und interdisziplinäre Reflexion von Forschungs- 
schwerpunkten, -lücken und -defiziten anregen. Der sorgfältige Einsatz szientometrischer Methoden ist also ein sinnvolles, aber nicht hinreichendes Hilfsmittel bei der schwierigen Erfassung und Beschreibung der Entwicklung von Forschungserträgen und damit ein erster deskriptiver Schritt dazu. Dieser Schritt soll hier für die Beiträge der Psychologie zur Bildungsforschung gemacht werden - nicht mehr, aber auch nicht weniger.

\section{Beitrag der Psychologie zur Bildungsforschung}

Warum wird nun eine disziplinspezifische szientometrische Perspektive für ein interdisziplinäres Feld wie die Bildungsforschung gewählt?

Zum einen existieren deutliche disziplinspezifische Unterschiede in den Publikationskulturen der an der interdisziplinären Bildungsforschung beteiligten Fächer (Knaupp et al., 2014; Kuhberg-Lasson et al., 2014). Der Psychologie (oder der psychologischen Bildungsforschung) wird zudem teilweise - etwa von Götz, Frenzel und Pekrun (2010, S. 71) - der Status einer «zentralen Grundlagenwissenschaft der Bildungsforschung» zugesprochen.

Zum anderen sollen hier Entwicklungslinien der Bildungsforschung in den letzten Dekaden beschrieben werden, die sich u.a. auf systematische Vergleiche der Bildungsforschung im deutschsprachigen Bereich mit Publikationen aus den angloamerikanischen Ländern beziehen. Als deutschsprachiger Bereich gelten dabei die sogenannten D-A-CH-L-L-Länder, also Deutschland [D] und Österreich $[\mathrm{A}]$ sowie die deutschsprachigen Teile der Schweiz [CH], von Lichtenstein [L] und Luxemburg [L]). Für entsprechende Datenbank-Recherchen ist nicht nur die eindeutige Identifikation der Publikationssprache notwendig, sondern vor allem die der Affiliation der Autorinnen und Autoren. Dies ist durchgängig nach dem Thesaurus of Psychological Index Terms der American Psychological Association (APA; Gallaher Tuleya, 2007) in den Fachdatenbanken PsycINFO und PSYNDEX mit dem Dokumentationsfeld «Institution»(IN) gesichert. PsycINFO ist die Fachdatenbank für die Psychologie und ihre Nachbardisziplinen mit angloamerikanischem Schwerpunkt (> $90 \%$ der Nachweise). PSYNDEX ist die Fachdatenbank für die Psychologie und ihre Nachbardisziplinen im deutschsprachigen Bereich (unter Einschluss englischsprachiger Publikationen aus dem deutschsprachigen Bereich).

\section{Ziele und Fragestellungen}

Zielsetzung ist ein szientometrisches Monitoring der Entwicklung des psychologischen Publikationsaufkommens zur Bildungsforschung insgesamt und zur Teilmenge der empirischen Bildungsforschung (vgl. hierzu etwa McElvany \& Holtappels, 2013) seit den 1950er Jahren im deutschsprachigen Bereich und in 
Publikationen aus den angloamerikanischen Ländern. Empirisch nachgegangen wird dabei u.a. die in den Wissenschaften allgemein und auch in der Bildungswissenschaft seit etwa zwei Dekaden virulente, kontrovers diskutierte Frage nach der Anglifizierung und Internationalisierung des Publikationswesens. Ferner soll das in der Bildungswissenschaft ebenfalls virulente Diskussionsthema ihrer empirischen Fundierung («Empirisierung») szientometrisch in ihrer Entwicklung analysiert werden. Durch das komparative Vorgehen zur Bildungsforschung in den angloamerikanischen versus deutschsprachigen Ländern werden Gemeinsamkeiten und Unterschiede in der Forschungsentwicklung und in den Forschungsschwerpunkten beschreibbar, die zur Reflexion der jüngeren Geschichte und der Gegenwart der Beiträge der Psychologie zur Bildungsforschung anregen können. Identifiziert werden sollen somit neben Schwerpunkten auch Lücken und Defizite in der Forschung und den Publikationen zu den psychologischen Grundlagen der Bildungsforschung und der Angewandten Bildungsforschung. Dies kann gerade auch für die bildungswissenschaftlichen Praktikerinnen und Praktiker von Interesse sein, von denen der Nutzen der Bildungsforschung für ihre Anwendungspraxis u.a. wegen anwendungs-irrelevanter Schwerpunkte und anwendungs-relevanter Lücken in Frage gestellt wird.

Unsere deskriptiv ausgerichteten Fragestellungen sind:

1. Wie hat sich seit den 1950er Jahren das psychologische Publikationsaufkommen zur Bildungsforschung und zu der Teilmenge der empirischen Bildungsforschung

a. im deutschsprachigen Bereich (nach PSYNDEX unter Einschluss englischsprachiger Publikationen) quantitativ entwickelt?

b. im angloamerikanischen Bereich (nach PsycINFO) quantitativ entwickelt?

c. Die Vergleiche von (a) und (b) werden in Feinanalysen für die Zeit von 1980 bis 2014 (in Fünf-Jahres-Intervallen) differenziert.

2. Wie hat sich von 1980 bis 2014 der Anteil englischsprachiger Veröffentlichungen aus der Psychologie im deutschsprachigen Bereich zur Bildungsforschung

a. für die gesamte Bildungsforschung entwickelt?

b. für die Teilmenge der empirischen Bildungsforschung entwickelt?

3. Wie gut und umfassend ist die Abdeckung der Publikationen aus der Psychologie im deutschsprachigen Bereich zur Bildungsforschung für die gesamte und für die Teilmenge der empirischen Bildungsforschung in PsycINFO, der Fachdatenbank mit angloamerikanischem Schwerpunkt (der häufig das Attribut «international» zugeschrieben wird)?

4. Welche inhaltlichen Schwerpunkte weisen die psychologischen Publikationen zur Bildungsforschung nach (a) PSYNDEX und (b) PsycINFO, also die aus den deutschsprachigen versus angloamerikanischen Ländern, auf? Spezifiziert werden dabei u.a. zudem die relativen Anteile von Veröffentlichungen zur empirischen Bildungsforschung (vergleichend für PSYNDEX und 
PsycINFO) und von englischsprachigen Publikationen (nur für PSYNDEX) für nach dem APA Thesaurus of Psychological Index Terms (Gallaher Tuleya, 2007; siehe auch PSYNDEX Terms; Leibniz-Zentrum für Psychologische Information und Dokumentation [ZPID], 2011) differenzierbaren

a. Teilgebieten der psychologischen Grundlagen der Bildungsforschung (wie etwa Lern-, Gedächtnis-, Denk-, Motivations- und Entwicklungspsychologie) und

b. Teilgebieten der Angewandten Bildungsforschung (wie etwa Unterrichtsmethoden und -programme, Lernen und Leistung, Lehren und Erziehen, Hochbegabtenpädagogik, Sonderpädagogik und Förderunterricht; vgl. hierzu etwa auch Götz et al., 2010).

\section{Methoden}

\section{Datenbasis}

Die Grundgesamtheit aller in den Fachdatenbanken für die Psychologie und ihre Nachbardisziplinen PSYNDEX und PsycINFO dokumentierten wissenschaftlichen Publikationen bildet die Datenbasis der Analysen. Aus dieser Grundgesamtheit werden die Stichproben der für die Bildungsforschung relevanten Nachweise mit identischen Recherchestrategien selegiert. Diese Grundgesamtheit der Literaturnachweise deckt auch ein breites Spektrum von Publikationen aus Nachbardisziplinen der Psychologie in den jeweiligen Überlappungsbereichen $\mathrm{ab}$.

PsycINFO ist die von der American PsychologicalAssociation (APA, Washington/ DC) produzierte, von der APA als «international» beworbene Fachdatenbank für Psychologie und ihre Nachbardisziplinen mit einem markanten anglo-amerikanischen Schwerpunkt (> 90 \% angloamerikanische Publikationen; < 2 \% Publikationen aus dem deutschsprachigen Bereich). Nachgewiesen werden JournalBeiträge, Monographien, Editionen, Dissertationen und wenige Buchbeiträge. Nach der Selbstbeschreibung reicht die Dokumentation bis 1806 zurück. De facto liegt eine umfassendere Dokumentation angloamerikanischer Publikationen seit dem Ende der 1970er Jahre vor. Dabei gab es im Übergang von der Papierversion der Psychological Abstracts zur digitalen Version PsycLit/PsycINFO (1975) für einige Jahre erhebliche Transferprobleme (mit nachhaltigen Dokumentationsmängeln). Zum Recherchezeitpunkt enthält die Datenbank ca. 4 Millionen Nachweise und ist etwa über OVID- und EBSCO-Plattformen in wissenschaftlichen Bibliotheken, http://www.apa.org/pubs/databases/psycinfo/ index.aspx oder www.MEDPILOT.de verfügbar. 
PSYNDEXplusTestfinder ist die vom Leibniz-Zentrum für Psychologische Information und Dokumentation (ZPID, Trier) produzierte Fachdatenbank für die Psychologie und ihre Nachbardisziplinen aus den deutschsprachigen Ländern (D-A-CH-L-L). Dokumentiert werden deutsch- und englischsprachige JournalBeiträge, Monographien, Editionen, Buchbeiträge, AV-Medien, Dissertationen und graue Literatur (exhaustiv ab dem Publikationsjahr 1980) sowie psychologische und pädagogische Testverfahren (ab 1945). Ebenso wie in PsycINFO umfasst die ausführliche Dokumentation in PSYNDEX u.a. seit 2006 die Affiliationen aller Autoren eines Beitrags, bis 2005 die Affiliation des Erstautors bzw. korrespondierenden Autors (vollständige Anschriften inklusive Institutsnamen). PSYNDEX enthält zum Recherchezeitpunkt ca. 300.000 Nachweise und ist etwa über OVID- und EBSCO-Plattformen in wissenschaftlichen Bibliotheken oder www.MEDPILOT.de und kostenfrei als Teil von PubPsych über www.pubpsych. de (open access) verfügbar.

\section{Recherche der Publikationsnachweise zur \\ Bi ldungs forschung}

Die Teilmenge der psychologischen Publikationen zur Bildungsforschung insgesamt in PsycINFO bzw. PSYNDEX wurde über die Recherche nach den folgenden Classification Codes (CC nach dem Thesaurus of Psychological Index Terms; Gallaher Tuleya, 2007) und deren Verknüpfung mit logischem «Oder» vorgenommen:

(1) Educational psychology $\left(\mathrm{CC}=35^{*}\right)$ or educational measurement $(\mathrm{CC}=$ 2227) für Literatur zur Angewandten Bildungsforschung,

(2) Cognitive processes, learning, memory, attention ( $\left.\mathrm{CC}=234^{*}\right)$, motivation, emotion $\left(\mathrm{CC}=236^{*}\right)$, developmental psychology $\left(C C=28^{*}\right)$ or learning disorders $(\mathrm{CC}=3253)$ für Publikationen zu den psychologischen Grundlagen der Bildungsforschung.

\section{Recherche der Publikationsnachweise zur empirischen Bi ldungs forschung}

Die Bestimmung der Untermenge psychologischer Publikationen zur empirischen Bildungsforschung an der Gesamtrecherche für die Bildungsforschung wurde über deren Kombination mit logischem «Und» für das Dokumentationsfeld Methodology/Study Type (MD nach dem Thesaurus of Psychological Index Terms; Gallaher Tuleya, 2007) mit folgenden Zuordnungen (OderVerbindungen) vorgenommen: empirical, experimental, longitudinal, qualitative empirical study, meta-analysis $\left(\mathrm{MD}=101^{*}\right)$, methodological study, assessment or intervention method description $\left(\mathrm{MD}=11^{*}\right)$ or literature review $(\mathrm{MD}=1310)$.

Mit dieser Recherchestrategie wird methodologisch ein umfassender Begriff von empirischer Bildungsforschung beachtet, der qualitative Studien (vgl. etwa Garz \& Blömer, 2010) ebenso wie quantitative (vgl. etwa Seel, Pirnay-Dummer, \& Ifenthaler, 2010) einbezieht. Aus der Teilmenge ausgeschlossen bleiben nicht- 
empirische Publikationen zur Bildungsforschung der Literaturgattungen study project, theoretical study, theoretical discussion, conceptual contribution, professional statement, textbook, handbook, self-help guide, comment, comment reply, bookl medialtest review, historical study, (auto)biography, laudation, obituary, professional policies/standards, discussion of servicelscience structures, directory, dictionary, glossary, bibliography, reprint, interview. Selbstverständlich sind diese aber in den Gesamtstichproben der psychologischen Publikationen zur Bildungsforschung nach PsycINFO und PSYNDEX berücksichtigt.

\section{Ergebnisse}

\section{Gesamtzahl psychologischer Publikationen zur Bildungsforschung}

Die Ergebnisse der auf die gesamten Datenbanken bezogenen Recherchen nach der Gesamtzahl psychologischer Veröffentlichungen zur Bildungsforschung und zur Teilmenge der empirischen Bildungsforschung sind in der Übersicht in Tabelle 1 zusammengestellt. Die absoluten Häufigkeiten $(f)$ bestätigen zunächst den bekannten sehr starken Zuwachs wissenschaftlicher Veröffentlichungen in der zweiten Hälfte des 20. Jahrhunderts. Dieser Zuwachs wurde vor allem durch die zunehmende Digitalisierung des Publikationswesens und das Internet im letzten Viertel des 20. Jahrhunderts weiter beschleunigt. Für die Anzahl psychologischer Beiträge zur gesamten Bildungsforschung sind aber weder für den deutschsprachigen noch für den angloamerikanischen Bereich überproportionale Zunahmen zu konstatieren, da die Zuwächse bei PSYNDEX (1978-2014) ebenso wie bei PsycINFO (1960-2014) statistisch bedeutsam und in ihrer Höhe essentiell mit der Entwicklung der Gesamtzahl aller dokumentierten psychologischen Publikationen korreliert sind (PSYNDEX: $r=.88$; PsycINFO: $r=.91$; beide $p<.01$ ). Die markante Zunahme psychologischer Beiträge zur Bildungsforschung seit den 1960- bzw. 1970er-Jahren ist also keine Besonderheit, sondern entspricht dem Anstieg psychologischer Publikationen generell (also unter Einschluss solcher zur Klinischen Psychologie, Wirtschaftspsychologie, zu anderen psychologischen Grundlagenfächern etc.).

Die für PsycINFO, also vor allem angloamerikanische Publikationen, festgestellten Häufigkeiten psychologischer Beiträge zur Bildungsforschung liegen etwa 10-fach höher als die für den deutschsprachigen Bereich ermittelten Werte nach PSYNDEX (siehe Tab. 1). Dies ist etwas weniger als sich für die Gesamtzahlen aller in PsycINFO versus PSYNDEX dokumentierten psychologischen Veröffentlichungen ergibt (insgesamt ca. 13-fach mehr Nachweise in PsycINFO als in PSYNDEX). 
Tabelle 1. Absolute Anzahl der psychologischen Publikationsnachweise zur Bildungsforschung und der Teilmenge zur empirischen Bildungsforschung nach PSYNDEX und PsycINFO für (1) den gesamten Zeitraum der Datenbanken und (2) für Neun-Jahres-Intervalle

\begin{tabular}{|c|c|c|c|}
\hline \multirow{2}{*}{$\begin{array}{l}\text { Psychologische } \\
\text { Fachdatenbank }\end{array}$} & \multirow{2}{*}{$\frac{\text { (1) Bildungsforschung a }}{\mathrm{f} \text { (absolut) }}$} & \multicolumn{2}{|c|}{ (2) empirische Bildungsforschung b } \\
\hline & & $\mathrm{f}$ (absolut) & relativer Anteil (\%) \\
\hline PSYNDEX 1945-2014 c & 72.457 & 31.604 & $43.6 \%$ \\
\hline$-1952-1960$ & 107 & 17 & $16 \%$ \\
\hline$-1961-1969$ & 201 & 23 & $11 \%$ \\
\hline$-1970-1978$ & 1.778 & 76 & $4 \%$ \\
\hline$-1979-1987$ & 11.016 & 1.701 & $15 \% \mathrm{c}$ \\
\hline$-1988-1996$ & 17.461 & 6.844 & $39 \%$ \\
\hline$-1997-2005$ & 18.489 & 9.453 & $51 \%$ \\
\hline$-2006-2014$ & 23.344 & 13.489 & $57,8 \%$ \\
\hline PsycINFO 1806-2014d & 715.750 & 399.838 & $55.9 \%$ \\
\hline$-1952-1960$ & 14.686 & 1.688 & $11 \%$ \\
\hline$-1961-1969$ & 24.330 & 5.931 & $24 \%$ \\
\hline$-1970-1978$ & 61.315 & 1.793 & $3 \% d$ \\
\hline$-1979-1987$ & 95.392 & 45.407 & $48 \%$ \\
\hline$-1988-1996$ & 109.655 & 75.561 & $69 \%$ \\
\hline$-1997-2005$ & 132.330 & 91.436 & $69 \%$ \\
\hline$-2006-2014$ & 237.373 & 174.591 & $73.6 \%$ \\
\hline
\end{tabular}

${ }^{a}$ Recherche (1) für die Classification Codes (CC) des APA-Thesaurus of Psychological Index Terms (Gallaher-Tuleya, 2007) für educational psychology $\left(C C=35^{*}\right)$, educational measurement $(C C=2227)$ sowie deren Grundlagenfächer cognitive processes, learning, memory, attention $\left(\mathrm{CC}=234^{*}\right)$, motivation, emotion $\left(C C=236^{*}\right)$, developmental psychology $\left(C C=28^{*}\right)$, learning disorders $(C C=3253)$ mit logischem «Oder».

b Kombination von Recherche (1) mit logischem «Und» für (a) PSYNDEX mit den Study Types (MD) für empirical, experimental, longitudinal, qualitative empirical studies, meta-analysis $\left(\mathrm{MD}=101^{*}\right)$, methodological study, assessment method description, intervention method description, manual (MD = $11^{*}$ ) oder literature review $(\mathrm{MD}=1310)$ bzw. für $(\mathrm{b})$ PsycINFO mit Methodology $(\mathrm{MD})$ für empirical, experimental, followup, longitudinal, prospective, retrospective study $\left(\mathrm{MD}=04^{*}\right)$, field study $(\mathrm{MD}=$ $\left.06^{*}\right)$, literature review $(\mathrm{MD}=08)$, meta analysis $(\mathrm{MD}=1200)$ oder quantitative study $(\mathrm{MD}=1800)$.

c PSYNDEX ist die ab PY $=1980$ exhaustive Fachdatenbank für die Psychologie aus den deutschsprachigen Ländern (D-A-CH-L-L), in der deutsch- und englischsprachige Fachliteratur und AV-Medien aus der Psychologie und ihren Nachbardisziplinen ab 1949 (exhaustiv ab 1980; auch digital) und psychologische Testverfahren ab 1945 dokumentiert werden (www.zpid.de).

d PsycINFO ist die internationale Fachdatenbank für Psychologie mit klarem anglo-amerikanischem Schwerpunkt (> $90 \%$ aller Nachweise; Nachweise z.B. aus dem deutschsprachigen Bereich: < $2 \%$ ) und mit umfangreicher Literaturdokumentation ab 1975 (seitdem digital mit mehrjährigen Umstellungsproblemen) sowie selektiver Dokumentation früherer Publikationen (www.apa.org/psycinfo/). 


\section{Psychologische Publikationen zur empirischen}

\section{Bildungsforschung}

Für den relativen Anteil empirischer psychologischer Beiträge zur gesamten Bildungsforschung - also psychologischer Publikationen zur empirischen Bildungsforschung - belegen die Angaben in der rechten Spalte von Tabelle 1 für PsycINFO ab den 1980er-Jahren, für PSYNDEX erst ab den 1990er-Jahren sehr starke Zuwächse. Nach diesem time lag von ca. zehn Jahren liegen die relativen Maximalwerte für den Anteil psychologischer Beiträge zur empirischen Bildungsforschung am Gesamt der psychologischen Beiträge zur Bildungsforschung im jüngsten Zeitintervall 2006-2014 für PsycINFO bei $74 \%$ und für PSYNDEX bei $58 \%$ (siehe Tab. 1). Festzustellen ist darüber hinaus, dass über $90 \%$ aller in PsycINFO und in PSYNDEX dokumentierten psychologischen Beiträge zur empirischen Bildungsforschung nach 1979 publiziert wurden. Da der wesentliche Entwicklungsschub psychologischer Beiträge zur Bildungsforschung somit seit den 1980er-Jahren aufgetreten ist, werden im Folgenden die Befunde von Feinanalysen für Fünf-Jahres-Intervalle ab 1980 dargestellt.

\section{Feinanalysen der Publikationshäufigkeiten von 1980 bis 2014}

Für die Zeit nach 1979 zeigt Abbildung 1 bis zur Jahrtausendwende einen relativ kontinuierlichen Anstieg der Anzahl aller psychologischen Publikationen zur Bildungsforschung und der zur Teilmenge der empirischen Bildungsforschung aus dem deutschsprachigen Bereich (nach PSYNDEX), der in den sogenannten Nullerjahren (nach dem Millennium) eine markante Beschleunigung erfährt. Für die entsprechenden angloamerikanischen Publikationszahlen (nach PsycINFO) ist die Zunahme bis kurz nach der Jahrtausendwende etwas schwächer ausgeprägt, um dann ebenfalls in den Nullerjahren sehr stark zu beschleunigen (siehe Abb. 2).

Für das Insgesamt der psychologischen Publikationen zur Bildungsforschung aus dem deutschsprachigen Bereich (nach PSYNDEX) sind in Abbildung 3 die Anteile deutsch- und englischsprachiger Veröffentlichungen differenziert. Deutlich wird, dass der allgemein für die Publikationszahlen zu beobachtende Anstieg vor allem auf englischsprachige Veröffentlichungen zurückzuführen ist. Diese haben insbesondere seit den Nullerjahren stark zugenommen, während simultan die Anzahl deutschsprachiger Beiträge stagniert. Entsprechend sind die Entwicklungskurven der absoluten Häufigkeiten zwischen (A) der Anzahl aller psychologischen Beiträge zur gesamten Bildungsforschung und (B) denen der deutschsprachigen statistisch nicht bedeutsam korreliert (nominal für die 35 Häufigkeitswerte von 1980 bis 2014: $r=.28$; $p>.10$ ). Ebenso ist dies bei den Entwicklungskurven für die Häufigkeiten zwischen (B) der Anzahl der deutschsprachigen und (C) der Anzahl der englischsprachigen Publikationen zur Bildungsforschung aus dem deutschsprachigen Bereich $(r=.23$; $\mathrm{p}>.10)$. Essentielle Höhe und statistische Signifikanz erreicht mit $r=.77(p<.01)$ nur der Zusammenhang zwischen (A) und (C). 


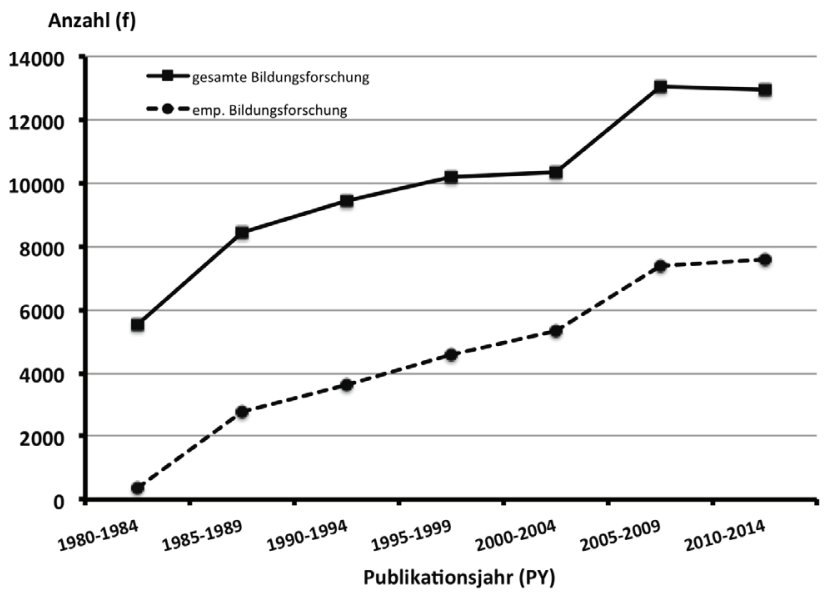

Abbildung 1: Anzahl aller psychologischen Publikationen zur Bildungsforschung und zur Teilmenge der empirischen Bildungsforschung nach PSYNDEX (unvollständig für 2014)

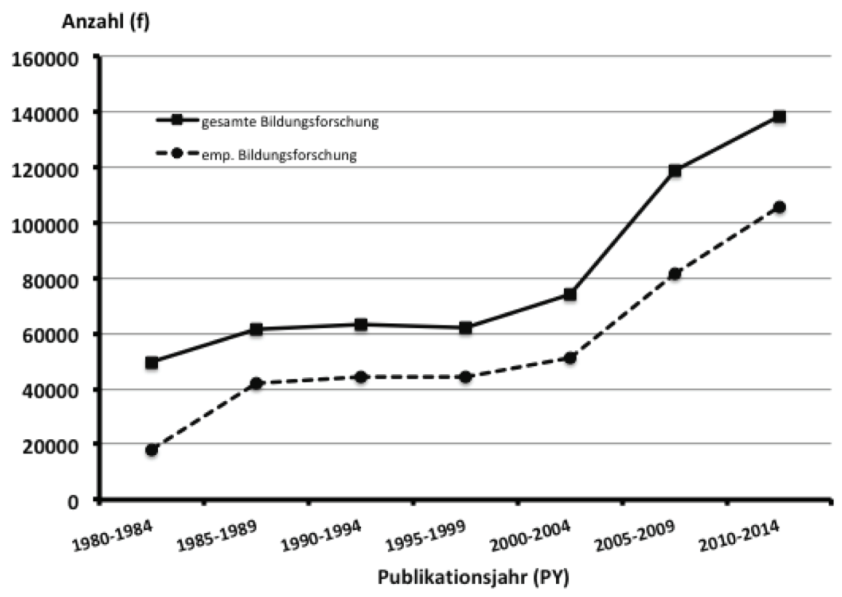

Abbildung 2: Anzahl aller psychologischen Publikationen zur Bildungsforschung und zur Teilmenge der empirischen Bildungsforschung nach PsycINFO (unvollständig für 2014)

Daraus folgt, dass die markante Zunahme von psychologischen Beiträgen zur Bildungsforschung im deutschsprachigen Bereich auf die Zunahme des englischsprachigen Anteils daran zurückzuführen ist. Bestätigt wird dies in Abbildung 4 anhand der Entwicklungskurven für die Anzahl englischsprachiger Publikationen (1.) zur Bildungsforschung insgesamt und (2.) zur empirischen Bildungsforschung aus dem deutschsprachigen Bereich (nach PSYNDEX). 


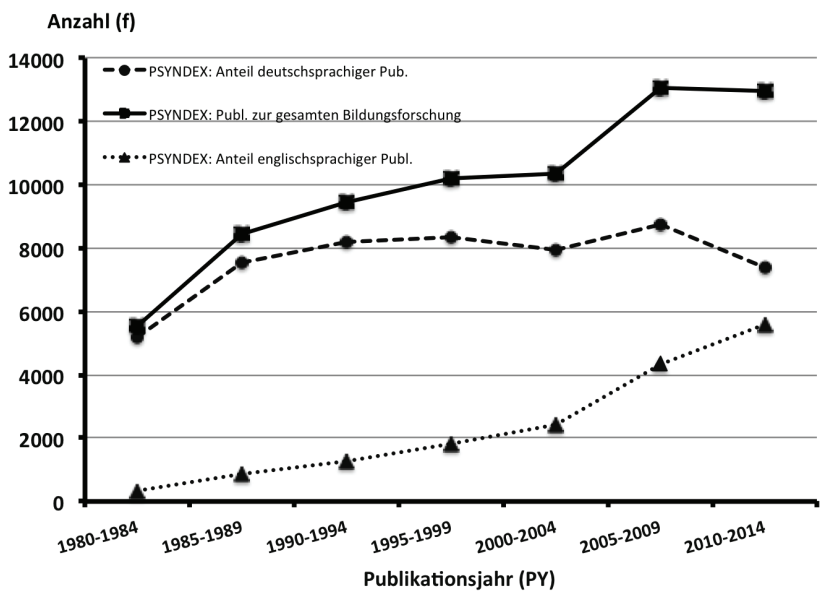

Abbildung 3: Anzahl aller psychologischen Publikationen zur gesamten Bildungsforschung sowie die deutsch-und englischsprachigen nach PSYNDEX (unvollständig für 2014)

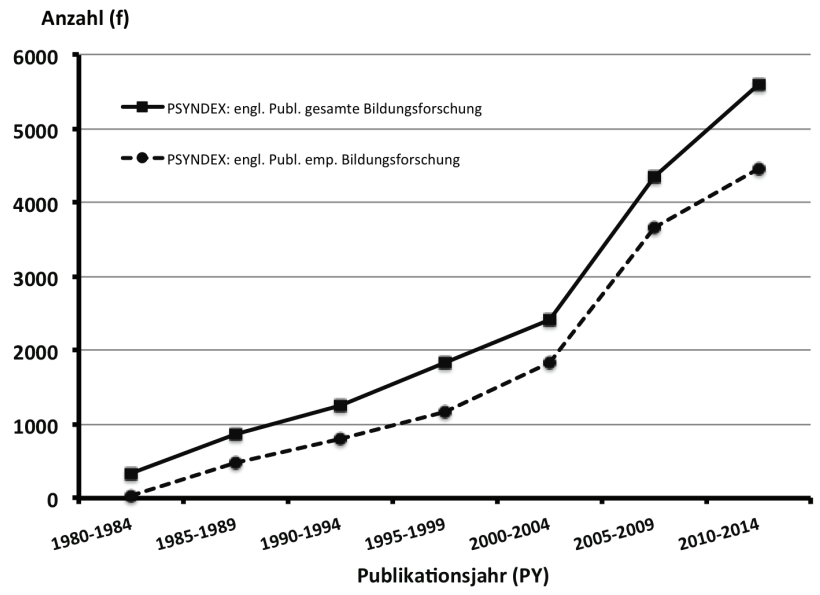

Abbildung 4: Anzabl aller englischsprachiger psychologischen Publikationen zur Bildungsforschung und zur Teilmenge der empirischen Bildungsforschung nach PSYNDEX (unvollständig für 2014) 


\section{Abdeckung psychologischer Publikationen zur Bildungsforschung aus dem deutschsprachigen Bereich in Psycinfo}

Die Repräsentanz der englischsprachigen Beiträge aus der Psychologie aus dem deutschsprachigen Bereich zur gesamten und zur Teilmenge der empirischen Bildungsforschung in der dominant angloamerikanischen Fachdatenbank PsycINFO liegt mit einigen Jahresschwankungen bei einer Abdeckung von rund $81 \%$. Zu konstatieren ist, dass in der Zeit von 1980 bis 2014 insgesamt $17 \%$ der englischsprachigen Beiträge zur gesamten psychologischen Bildungsforschung (das sind insgesamt immerhin 2.846 Publikationen oder 81 Publikationen pro Erscheinungsjahr; siehe Abbildung 5) und $20 \%$ der zur Teilmenge der empirischen psychologischen Bildungsforschung (2.439 englischsprachige Publikationen oder 70 pro Jahr; siehe Abbildung 6) aus den D-A-CH-L-L-Ländern nicht in PsycINFO Eingang gefunden haben. Damit ist selbst der englischsprachig publizierte Forschungsertrag zur psychologischen Bildungsforschung aus dem deutschsprachigen Bereich in PsycINFO unterrepräsentiert. Betroffen davon sind nicht nur englischsprachige Journal-, sondern vor allem auch englischsprachige Buchbeiträge und Dissertationen aus den D-A-CH-L-L-Ländern.

Markanter wird die Unterrepräsentation der psychologischen Bildungsforschung aus den D-A-CH-L-L-Ländern, wenn die Abdeckung der in PSYNDEX dokumentierten deutschsprachigen Publikationen in PsycINFO, einer nach der Selbstbeschreibung «internationalen» Fachdatenbank, betrachtet wird (siehe Abbildung 7). Die wenigen in PsycINFO dokumentierten deutschsprachigen Publikationen zur Bildungsforschung entstammen ausschliesslich wenigen psychologischen Fachzeitschriften, deren Mehrheit im übrigen inzwischen anglifiziert wurde (vgl. Krampen, Huckert, \& Schui, 2012). Im Unterschied zu PSYNDEX bietet die Recherche in PsycINFO zudem keinen Zugang zu Monographien, Editionen, Dissertationen, Buchbeiträgen und Testverfahren aus dem deutschsprachigen Bereich. Dadurch finden diese Literaturgattungen bei szientometrischen Evaluationen und beim Monitoring des Forschungsertrages von Personen und Institutionen aus den D-A-CH-L-L-Ländern anhand von PsycINFO ebenso wie anhand des Web of Science (WoS) keine Beachtung. 


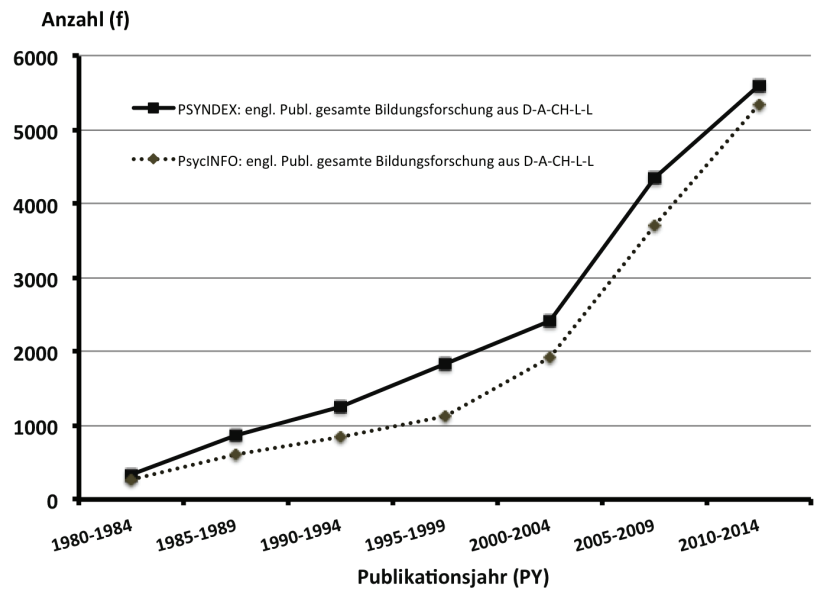

Abbildung 5: Anzahl englischsprachiger psychologischen Publikationen zur Bildungsforschung aus dem deutschsprachigen Bereich (D-A-CH-L-L) nach PSYNDEX versus PsycINFO(unvollständig für 2014)

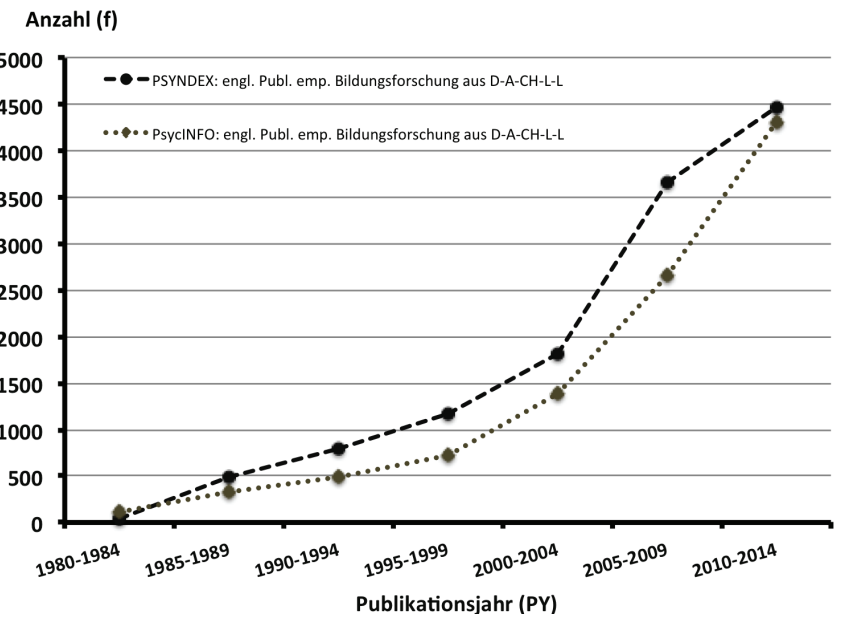

Abbildung 6: Anzahl englischsprachiger psychologischen Publikationen zur empirischen Bildungsforschung aus dem deutschsprachigen Bereich (D-A-CH-L-L) nach PSYNDEX und PsycINFO(unvollständig für 2014) 


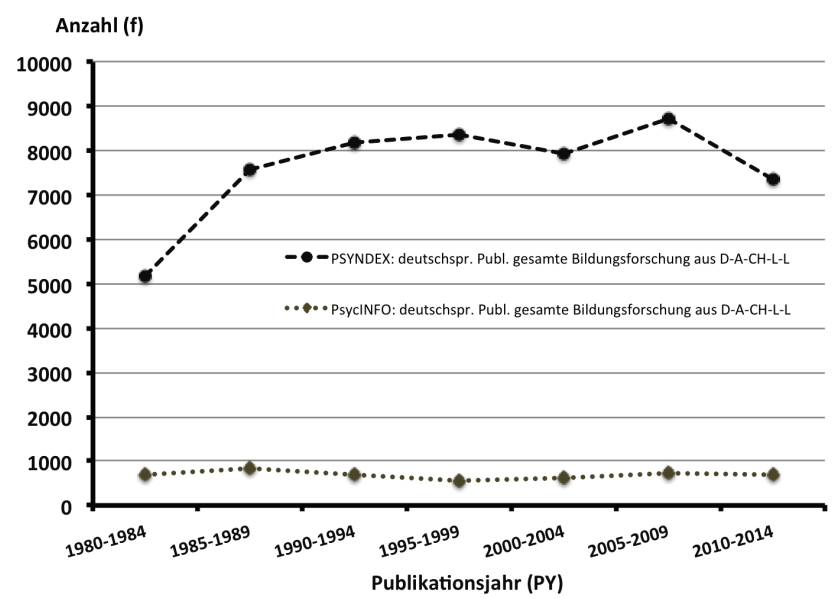

Abbildung 7: Anzahl deutschsprachige psychologischen Publikationen zur gesamten Bildungsforschung aus dem deutschsprachigen Bereich (D-A-CH-L-L) und PSYNDEX versus PsycINFO(unvollständig für 2014)

\section{Inhaltliche Schwerpunkte der psychologischen \\ Bildungsforschung}

Für die Gesamtzahlen der Nachweise zur psychologischen Bildungsforschung in PSYNDEX und in PsycINFO sind in Tabelle 2 die absoluten Häufigkeiten sowie die relativen Anteile englischsprachiger Publikationen (für PSYNDEX) und die der empirischen Bildungsforschung (für PSYNDEX und für PsycINFO) für verschiedene Teilgebiete der angewandten psychologischen Bildungsforschung und der psychologischen Grundlagen der Bildungsforschung zusammengestellt. Dies folgt den Unter-Klassifikationen [CC] nach dem Thesaurus of Psychological Index Terms (Gallaher Tuleya, 2007). Zu beachten ist dabei, dass aufgrund von indizierten Doppel-Klassifikationen einzelner Publikationen - also z.B. die Klassifikation einer Publikation zu den Grundlagen des Lernens $\left(C C=234^{*}\right)$ in der Grundschule nach einem bestimmten Unterrichtsprogramm $(\mathrm{CC}=3530)$ wie in Klammern angegeben zweifach klassifiziert wird - die absoluten Häufigkeitswerte nicht den Angaben in Tabelle 1 entsprechen.

Die Ergebnisse zeigen, dass sowohl im deutschsprachigen Bereich als auch im angloamerikanischen die Anzahl der Publikationen zu den psychologischen Grundlagen der Bildungsforschung höher liegt als die zur Angewandten Bildungsforschung. Dies gilt ebenso für den jeweiligen Anteil der Beiträge zur empirischen Bildungsforschung. Bei den Grundlagenstudien dominieren Beiträge aus der Entwicklungspsychologie (cognitive \& psychosocial development) sowie der Kognitions-, Lern- und Aufmerksamkeitspsychologie (cognitive processes, learning \& attention). $\mathrm{Zu}$ den motivations- und emotionspsychologischen Grundlagen von Bildung (motivation \& emotion) wird dagegen selten 
publiziert. Dies gilt gleichermassen für die Bildungsforschung aus dem deutschsprachigen (PSYNDEX) und dem angloamerikanischen Bereich (PsycINFO). $\mathrm{Zu}$ Lernstörungen (learning disorders) wird dagegen in der Bildungsforschung aus dem deutschsprachigen Bereich wenig, in der aus dem angloamerikanischen sehr viel publiziert. Englischsprachig werden aus dem deutschsprachigen Bereich vor allem Arbeiten zu den kognitionspsychologischen (51\% Englisch) sowie motivations- und emotionspsychologischen Grundlagen (34\%) von Bildungsprozessen publiziert.

In der Angewandten Bildungsforschung liegen die Schwerpunkte psychologischer Beiträge übereinstimmend für PSYNDEX und PsycINFO eindeutig bei den Themen Unterrichtsmethoden und -programme (teaching methods \& programs) sowie Lernen und Leistung (academic learning \& achievement), die bei deutlich geringeren absoluten Grundhäufigkeiten relativ betrachtet im deutschsprachigen Bereich ebenso etwas häufiger thematisiert werden als im angloamerikanischen wie die Themen Sonderpädagogik und Förderunterricht (special \& remedial education), Hochbegabtenpädagogik (gifted \& talented) sowie pädagogisch-psychologische Messung und Tests (educational measurement). Der englischsprachige Anteil der Publikationen aus den D-A-CH-L-L-Ländern liegt bei den Themenbereichen Hochbegabtenpädagogik (16\% Englisch; gifted \& talented) sowie Lernen und Leistung (14\%; academic learning \& achievement) am höchsten (siehe Tab. 2).

\section{Diskussion und Fazit}

Die Entwicklung der relativen Publikationszahlen zur gesamten psychologischen Bildungsforschung aus den deutschsprachigen Ländern ist nach den dargestellten szientometrischen Befunden bei markant geringerer absoluter Grundgesamtheit mit der Entwicklung entsprechender angloamerikanischer Publikationszahlen seit den 1950er-Jahren vergleichbar. Der deutliche Anstieg ist zunächst keine Besonderheit, sondern entspricht gut dem Zuwachs psychologischer Publikationen generell. Dies ist ähnlich zum markant angestiegenen Publikationsaufkommen in anderen Wissenschaften in der zweiten Hälfte des 20. Jahrhunderts. Dieser häufig als «Publikationsboom» benannte Anstieg kann sowohl auf die gestiegenen ökonomischen und finanziellen Ressourcen von Forschung und (universitärer) Bildung (und die dahinter stehenden gesellschaftlichen, politischen und wirtschaftlichen Interessen) als auch auf die Digitalisierung und das Internet im letzten Viertel des 20. Jahrhunderts zurückgeführt werden.

Für die Teilmenge der psychologischen Beiträge zur empirischen Bildungsforschung sind allerdings für Publikationen aus den angloamerikanischen Ländern ab den 1980er-Jahren überproportionale Zuwächse festzustellen. Ab den 1990erJahren - also mit einer zeitlichen Verzögerung von etwa zehn Jahren - gilt dies ebenfalls für die deutschsprachigen Länder. Seit den 1980ern (angloamerikanische 
Publikationen) bzw. 1990ern (deutschsprachiger Bereich) ist die Mehrheit aller psychologischen Beiträge zur Bildungsforschung empirisch geworden. Dies ist besonders deutlich seit den Nullerjahren nach dem Millennium zu beobachten. Für den deutschsprachigen Bereich gilt dabei überdies, dass der starke Zuwachs der psychologischen Beiträge zur Bildungsforschung insgesamt alleine auf den starken Anstieg englischsprachiger empirischer Veröffentlichungen zurückzuführen ist, während die Anzahl deutschsprachiger Beiträge stagniert.

Diese «Empirisierung» der psychologischen Bildungsforschung (und auch ihre «Anglifizierung» bei den Publikationen aus dem deutschsprachigen Bereich) kann bislang kaum allein auf die Publikation der Erträge internationaler und nationaler Vergleichsstudien - also der Befunde von large-scale assessments schulischer Leistungen - zurückgeführt werden. Publikationen zur psychologischen Bildungsforschung aus solchen Projekten verfügen mit insgesamt 743 in PSYNDEX für 1995 bis 2014 nachgewiesenen psychologischen Publikationen zur empirischen Bildungsforschung nur über einen Anteil von $3 \%$. Davon sind $11 \%(n=79)$ englischsprachig, von denen viele methodischen oder methodologischen Fragen in internationalen Kooperationen nachgehen.

Gleichwohl sind diese englischsprachigen Publikationen aus internationalen und nationalen Vergleichsstudien zur empirischen Bildungsforschung aus den D-A-CH-L-L-Ländern ein Beispiel für die Themenbereiche, mit denen internationale Sichtbarkeit erreicht werden kann. Neben den Beiträgen zum Bereich «Lernen und Leistung» werden vor allem auch solche zu den kognitions-, motivations- und emotionspsychologischen Grundlagen von Bildungserfolg sowie zu psychologischen Aspekten der Hochbegabtenpädagogik erfolgreich in englischsprachigen Fachmedien platziert.

Komparativ sind dagegen für die psychologische Bildungsforschung aus den deutschsprachigen Ländern geringere Forschungs- bzw. Publikationsaktivitäten bei Themen wie "Pädagogisches Personal (Lehrer, Erzieher etc.)», "Lehrmethoden und Unterrichts-/Bildungsprogramme», «Interaktion, adjustment und Einstellung von Lernenden» sowie den "Grundlagen von Lernstörungen» festzustellen. Diese Themenbereiche werden in Publikationen aus den angloamerikanischen Ländern (nach PsycINFO) nicht nur absolut, sondern vor allem auch relativ betrachtet häufiger thematisiert und empirisch analysiert. Hier scheinen Defizite in der Bildungsforschung aus den deutschsprachigen Ländern zu bestehen, die kaum auf kulturelle Unterschiede zwischen den angloamerikanischen und den deutschsprachigen Bereichen zurückgeführt werden können. Die Implikationen für die Forschungspolitik und -planung in der Bildungswissenschaft liegen mit den Notwendigkeiten einer Forschungsförderung gerade in diesen Bereichen zur Verkleinerung dieser weissen Flecken auf der Hand. Dabei sollten - als weitere Implikation - theoretisch fundierte empirische Forschungsprojekte besondere Beachtung finden, damit der Anschluss an die internationale, hier angloamerikanische Bildungsforschung nicht verloren geht, sondern verbessert wird. 
Trotz der Zunahme auch englischsprachiger empirischer Beiträge zur psychologischen Bildungsforschung aus den deutschsprachigen Ländern bleibt deren unvollständige Abdeckung in sogenannten «internationalen» wissenschaftlichen Datenbanken wie PsycINFO, die de facto einen ausgeprägten angloamerikanischen Schwerpunkt aufweisen, bedenkenswert. Im Vergleich zur Fachdatenbank für die Psychologie und ihre Nachbardisziplinen im deutschsprachigen Bereich PSYNDEX und zur internationalen Fachdatenbank PubPsych mit europäischem Schwerpunkt fehlen in PsycINFO 17 \% der psychologischen Beiträge zur gesamten und $20 \%$ der Beiträge zur empirischen Bildungsforschung aus den D-A-CH-L-L-Ländern. Diese sind also bei Verwendung der vermeintlich internationalen Datenbank PsycINFO nicht nachgewiesen und werden damit weder in Literaturrecherchen noch in darauf basierenden Evaluationen sichtbar. Bei deutschsprachigen Publikationen zur psychologischen Bildungsforschung sind sogar $90 \%$ in PsycINFO nicht dokumentiert (coverage: $10 \%$ ). Nicht nur eine Vielzahl deutschsprachiger Journal-Beiträge, sondern vor allem auch alle deutschsprachigen Monographien, Editionen, Buchbeiträge und Testverfahren zur psychologischen Bildungsforschung bleiben unberücksichtigt. Ebenso verhält es sich mit dem coverage nicht-angloamerikanischer Publikationen bei anderen vermeintlich internationalen Ressourcen wie etwa Web of Science (WoS) und Scopus.

Daraus resultieren Implikationen auf individueller und institutioneller Ebene: Wollen Forscherinnen und Forscher der Bildungswissenschaft aus dem deutschsprachigen Bereich international sichtbar und damit kooperations- und anschlussfähig bleiben, so sind sie gefordert, vermehrt englischsprachig zu publizieren. Nur so kann in internationale Datenbanken Eingang gefunden werden. Wissenschaftspolitisch kann dies etwa durch darauf bezogene Fördermittel für englischsprachiges Lektorieren u.ä. - etwa auch im Rahmen der Drittmittelförderung - unterstützt werden. Wissenschaftspolitik und -management sind überdies gefordert, nicht-englischsprachige, ggf. auch europäische wissenschaftliche Fachdatenbanken (wie etwa FIS Bildung, PSYNDEX, PubPsych, SOLIS) zu erhalten bzw. auszubauen. Diese sind nicht nur wegen kultureller, ggf. auch nationaler Spezifika in Bildungs-, Rechts-, Gesundheitssystemen etc., sondern generell ergänzend zu den zurzeit dominant angloamerikanischen Datenbanken notwendig. Ihre über die wissenschaftliche Funktion hinausgehende gesellschaftspolitische Aufgabe ist es, nicht-englischsprachige wissenschaftliche Publikationen einem breiteren Publikum, neben den Wissenschaften selbst auch der Berufspraxis, der Aus-, Fort- und Weiterbildung, den Massenmedien, der interessierten Öffentlichkeit und - nicht zuletzt - auch grossen Teilen der Wissenschaftspolitik und des Wissenschaftsmanagements unmittelbar zugänglich zu erhalten.

Limitationen unserer Analysen sind dem quantitativen Ansatz der Szientometrie geschuldet. Forschungsschwerpunkte und -entwicklungen können ebenso wie Forschungslücken und -defizite szientometrisch für grössere Zeiträume 
beschrieben werden. Die Befunde bedürfen aber zusätzlich der fachlichen, inhaltlichen und gesellschaftlichen, also der qualitativen Deutung und Bewertung. So können etwa komparativ zur angloamerikanischen Bildungsforschung festgestellte quantitative Forschungslücken der Bildungsforschung im deutschsprachigen Bereich gezielt Anlass dazu geben, die vorliegende (dann überschaubarere) angloamerikanische Fachliteratur inhaltlich - mithin qualitativ - zu rezipieren, um Anregungen für die eigene Forschung zu bekommen. Auch die Übertragbarkeit angloamerikanischer Befunde auf Europa und andere Regionen dieser Welt sollte dabei immer wieder reflektierend in Frage stehen (vgl. dazu etwa Arnett, 2008, 2009). Zusätzlich können qualitativ rezipierend die bildungswissenschaftlichen Themenbereiche mit höheren Anteilen englischsprachiger Publikationen dabei von Nutzen sein, zu erkennen, an welchen Forschungsbereichen international Interesse besteht, bei welchen international Forschungs- und Publikationslücken existieren sowie wo und bei wem Anschluss- und Kooperationsfähigkeit für eigene Forschungsinteressen bestehen könnte.

\section{Anmerkung}

1 Die vorliegende Arbeit wurde im Rahmen des Leibniz-Forschungsverbundes «Bildungspotentiale» (LERN) verfasst.

\section{Literatur}

Arnett, J. J. (2008). The neglected 95\%: Why American psychology needs to become less American. American Psychologist, 63(7), 602-614.

Arnett, J. J. (2009). The neglected 95\%: A challenge to psychology's philosophy of science. American Psychologist, 64(6), 571-574.

Bühler, K. (1927). Die Krise der Psychologie. Jena: Gustav Fischer (Nachdruck [2000]. Weilerswist-Metternich: Velbrück). Verfügbar am 01.08.2018 unter https:// nasepblog. files.wordpress.com/2012/08/buehler-karl-die-krise-der-psychologie-kantstudien-31-1926.pdf

Felt, U., Nowotny, H., \& Taschwer, K. (1995). Wissenschaftsforschung. Frankfurt/Main: Campus.

Gallaher Tuleya, L. (Ed.). (2007). Thesaurus of psychological index terms (11 $1^{\text {th }} \mathrm{ed}$.). Washington, DC: American Psychological Association. Verfügbar am 01.08.2018 unter http://www. apa.org/pubs/databases/training/thesaurus.aspx

Garz, D., \& Blömer, U. (2010). Qualitative Bildungsforschung. In R. Tippelt \& B. Schmidt (Hrsg.). (2010). Handbuch Bildungsforschung (3. Aufl., S. 571-588). Wiesbaden: VS Verlag für Sozialwissenschaften.

Götz, T., Frenzel, A. C., \& Pekrun, R. (2010). Psychologische Bildungsforschung. In R. Tippelt \& B. Schmidt (Hrsg.). (2010). Handbuch Bildungsforschung (3. Aufl., S. 71-92). Wiesbaden: VS Verlag für Sozialwissenschaften.

Knaupp, M., Schauffler, S., Hofbauer, S., \& Keiner, E. (2014). Education research and educational psychology in Germany, Italy and the United Kingdom: An analysis of scholary journals. Schweizerische Zeitschrift für Bildungswissenschaften, 36, 83-108. Verfügbar am 01.08.2018 unter http://www.pedocs.de/volltexte/2015/10791/pdf/ SZBW_2014_1_ Knaupp_ua_Education_Research_and_Educational_Psychology.pdf

Köhler, E. (1929). Die Krise der Psychologie und die Pädagogik. Schulreform - Pädagogische 
Monatsschrift, 8(8/9), 40-9-427. Verfügbar am 01.08.2018 unter https://portal.dnb.de/ opac. htm? method=simpleSearch\&cqlMode=true\&query=idn\%3D574389229

Krampen, G. (2013). Zur Klärung des Stellenwerts szientometrischer Methoden in der Wissenschaftsevaluation aus psychologischer Sicht. Zeitschrift für Evaluation, 12(1), 79-102. Verfügbar am 01.08.2018 unter http://www.waxmann.com/ index. php\%3Fid\%3Dzeitschriftendetails\%26no_cache\%3D1\%26tx_p2waxmann_ pi1\%255Bid_zeitschrift\%255D\%3DZEI1007\%26tx_p2waxmann_pi1\%255Bid_ ausgabe\%255D\%3DAUG100172\%26tx_p2waxmann_pi1\%255Bshow\%255D\%3Dsho wAusgabeInhaltsangabe

Krampen, G., \& Montada, L. (2002). Wissenschaftsforschung in der Psychologie. Göttingen: Hogrefe.

Krampen, G., Huckert, T., \& Schui, G. (2012). Impact of anglicizing former Germanlanguage psychology journals on authorships and citation frequencies. European Psychologist, 17(3), 190-198. Verfügbar am 01.08.2018 unter http://econtent.hogrefe. com/doi/ abs/10.1027/1016-9040/a000074

Kuhberg-Lasson, V., Singleton, K., \& Sondergeld, U. (2014). Publikationscharakteristika im interdisziplinären Feld der Bildungsforschung. Journal for Educational Research Online, 6(3), 134-155. Verfügbar am 01.08.2018 unter http://www.pedocs.de/volltexte/2014/ 9691/pdf/JERO_2014_3_KuhbergLasson_Singleton_Sondergeld_Publikationscharakteristika.pdf

McElvany, N., \& Holtappels, H. G. (Hrsg.). (2013). Empirische Bildungsforschung: Theorien, Methoden, Befunde und Perspektiven - Festschrift für Wilfried Bos. Münster: Waxmann.

Seel, N. M., Pirnay-Dummer, P., \& Ifenthaler, D. (2010). Quantitative Bildungsforschung. In R. Tippelt \& B. Schmidt (Hrsg.), Handbuch Bildungsforschung (3. Aufl., S. 551-570). Wiesbaden: VS Verlag für Sozialwissenschaften.

Tippelt, R., \& Schmidt, B. (Hrsg.). (2010). Handbuch Bildungsforschung (3. Aufl.). Wiesbaden: VS Verlag für Sozialwissenschaften.

ZPID - Leibniz-Zentrum für Psychologische Information und Dokumentation. (Hrsg.). (2011). PSYNDEX Terms (9. Aufl.). Trier: ZPID. Verfügbar am 01.08.2018 unter http:// www. zpid.de/pub/info/PSYNDEXterms2011.pdf

Schlagworte: Bildungsforschung, Psychologie, empirische Bildungsforschung, Pädagogische Psychologie, Szientometrie, Geschichte der Bildungswissenschaften 


\section{Suivi des apports de la psychologie à la recherche interdisciplinaire en éducation: analyse scientométrique comparative de la recherche en contextes germanophones et angloanglophones}

\section{Résumé}

Les apports des publications spécialisées dans le domaine de la psychologie à la recherche en éducation sont examinés dans le cadre d'une analyse scientométrique. Cette spécification s'avère nécessaire à l'étude de l'évolution du nombre de publications dans le domaine de la psychologie de l'éducation en général en comparaison au domaine plus particulier de la recherche empirique en éducation, dans les pays germanophones et anglo-américains. Pour ce faire, des indications sur les auteur-e-s et leur affiliation des auteur-e-s sont indispensables. Ces indications ne figurent pas, ou uniquement de façon sélective, dans les banques de données spécialisées, alors qu'on les trouve de manière constante dans les banques de données du domaine de la psychologie (PSYNDEX et PsycINFO). Les résultats présentent le développement des publications psychologiques ayant pour thème la recherche en éducation et, en particulier, la recherche empirique en éducation (a) dans les pays germanophones et (b) anglo-américains ainsi que (c) la comparaison entre les deux. La diffusion des publications renvoie à une période allant de 1945 à 2014 alors qu'une analyse détaillée est effectuée pour la période allant de 1980 à 2014.

Mots-clés: Recherche en éducation, psychologie, recherche empirique, psychologie pédagogique, scientométrie, histoire des sciences de l'éducation

\section{Monitoraggio del contributo della psicologia nella ricerca interdisciplinare sulla educazione: Analisi scienza-metrica della ricerca nei paesi di lingua tedesca versus quelli di lingua inglese}

\section{Riassunto}

In una prospettiva interdisciplinare viene eon analizzato a livello scienza-metrico il contributo di quelle specifiche pubblicazioni di psicologia riguardanti l'educazione. Questa limitazione è necessaria per lo sviluppo del numero di pubblicazioni relative alla psicologia dell'educazione in generale e quelle relative al sottoinsieme della ricerca empirica sull'educazione sviluppate nei paesi di lingua tedesca che vengono messe a confronto con quelle di lingua inglese. Per questo sono importanti le indicazioni sugli autori/autrici e le loro affiliazioni, che in molte banche dati specialistiche non sono presenti o soltanto in modo selettivo, mentre sono normalmente disponibili in quelle relative alla psicologia (PSYNDEX, PsycINFO). I dati si riferiscono allo sviluppo del volume 
delle pubblicazioni psicologiche sull'educazione e sul sottoinsieme della ricerca empirica sull'educazione (a) in lingua tedesca e (b) in lingua inglese così come (c) il loro confronto. Viene descritta la diffusione delle pubblicazioni per gli anni 1945-2014 e un'analisi dettagliata per gli anni 1980-2014.

Parole chiave: Ricerca sull'educazione, psicologia, ricerca empirica sull'educazione, psicologia pedagogica, scienza-metrica, storia di scienze dell'educazione

\section{Monitoring of psychology's contribution to interdisciplinary educational research: Scientometric analyses of research in the German-speaking versus Anglo-American countries}

\section{Summary}

The contribution of psychological publications to interdisciplinary educational research is monitored scientometrically. Objectives refer to comparisons of publication trends (1945-2014) in Anglo-American publications (PsycINFO) and these from the German-speaking countries (PSYNDEX). Databases include continuously the affiliation of authors. Results refer to the development of psychological publications concerning educational research and of the subset of these concerning empirical educational research (a) in the Germany-speaking countries (including English publications), (a) in Anglo-American psychological publications and (c) comparisons of both. Psychological contributions to educational research increase since the 1980s significantly in the Anglo-American and with a time lag of ten years in the German-speaking countries. Both show very strong increases of psychological publications on empirical educational research after the millennium.

Keywords: Educational research, psychology, empirical research methods, educational psychology, scientometrics, history of educational sciences 
Günter Krampen, Prof. Dr., Studium der Psychologie, Germanistik, Anglistik und Soziologie; Dr. phil. 1980, Dr. rer.nat.habil 1985, PsychTh 1999; Prof. em. für Psychologie an der Universität Trier, Direktor Emeritus des LeibnizZentrums ZPID; Forschungsgebiete: Pädagogische und Klinische Psychologie, Geschichte der Psychologie, Wissenschaftsforschung

Leibniz Institute for Psychology Information ZPID, University of Trier, D-54286 Trier,

E-Mail: krampen@uni-trier.de

Marcus Hasselhorn, Prof. Dr., Studium der Psychologie; Dr. phil. 1986, Habilitation 1993; Prof. für Psychologie mit dem Schwerpunkt „Bildung und Entwicklung " an der Goethe-Universität Frankfurt; Direktor des Leibniz-Institutes DIPF; Forschungsgebiete: Frühe Bildung, Lernstörungen, Entwicklung individueller Voraussetzungen erfolgreichen Lernens

German Institute for International Educational Research DIPF, Schloßstr. 29, D-60486 Frankfurt am Main

E-Mail: hasselhorn@dipf.de 